\title{
Cooccurrence of Multilocular Cystic Renal Cell Carcinoma and High-Grade Urothelial Carcinoma in Bladder
}

\author{
Seyma Ozkanlia, b, Elvan Atsatan Turfanda ${ }^{\mathrm{a}}$, Tulay Zenginkinet ${ }^{\mathrm{a}}$, Abdullah Aydin ${ }^{\mathrm{a}}$
}

\begin{abstract}
Multilocular cystic renal cell carcinoma (MCRCC) is a rare subtype of RCC. As we know, there are not any cases with the cooccurrence of MCRCC and urothelial carcinoma in the literature. Here, we report a case of a 57-year-old man who was diagnosed with gross hematuria and dysuria. To our knowledge, this case report is the first one that analyzes these two tumors together.
\end{abstract}

Keywords: Urinary and genital tract disorders; Renal system; Carcinoma; Bladder

\section{Introduction}

Multilocular cystic renal cell carcinoma (MCRCC) is a rare subtype of RCC. There are not any cases with the cooccurrence of MCRCC and urothelial carcinoma in the Englishwritten literature. Here, we present a case of a 57-year-old man diagnosed with gross hematuria and dysuria. To our knowledge, this case report is the first one that focuses on these two tumor types together.

\section{Case Report}

A 57-year-old male patient with a history of 2 years of diabetes mellitus (DM), and 80-pack-year history of cigarette smoking applied to our clinic with gross hematuria, dysuria, and urgency. The patient had normal blood pressure and was febrile. While urinalysis was negative for nitrite and leukocyte esterase, it was $2+$ for protein and $3+$ for blood, and there were

Manuscript accepted for publication October 13, 2014

aDepartment of Pathology, Goztepe Training and Research Hospital, Istanbul Medeniyet University, Istanbul, Turkey

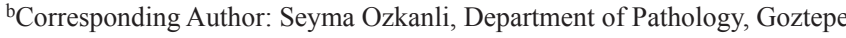
Training and Research Hospital, Istanbul Medeniyet University, Istanbul,

Turkey. Email: seymaozkanli@gmail.com

doi: http://dx.doi.org/10.14740/jmc1801w
3 - 6 leucocytes and too many erythrocytes to count per area in magnification. Urinary culture was sterile. Magnetic resonance imaging (MRI) showed a $5 \times 3 \times 3 \mathrm{~cm}$ multilocular cystic lesion in the right kidney's upper pole that looked hyperintense in T1 images (Fig. 1). On the right anterolateral wall of the bladder, there was an increase in wall thickness that reached into the bladder and was $25 \times 19 \times 35 \mathrm{~mm}$ in size (Fig. 2). Concurrent laparoscopic right-partial nephrectomy and transurethral resection (TUR) were performed on the patient.

\section{Partial nephrectomy}

Partial nephrectomy material, which was $5 \times 3 \times 2 \mathrm{~cm}$ in size, capsular and nodular in appearance, had a fatty tissue. Incision revealed many cysts that were $0.4-1 \mathrm{~cm}$ in size and that deposited hemorrhagic fluid. The lesion that the cysts formed was capsular and had regular borders (Fig. 3). Histopathological analysis revealed a tumor consisting of cells with clear cytoplasm and small hyperchromatic nucleuses, which paved the septas, and forming small papillary structures at some places and increasing ingradation in others (Fig. 4, 5).

\section{Bladder TUR specimen}

TUR material $(4 \mathrm{cc})$ was analyzed. Microscopic examination showed high-grade urothelial carcinoma which invaded the bladder wall and that had cells with pleomorphic, large atypical nucleuses and eosinophilic cytoplasm, and that had a high number of mitosis. The tumor had infiltrated muscle tissue. High-grade invasive urothelial carcinoma was regarded as pT2 (Fig. 6). The patient is still alive and he is followed up semi-annually.

\section{Discussion}

The widely accepted definition of multiple primary malignancies was made by Warren and Gates [1]. According to this, each tumor should meet definitive malignancy criteria, one should be different from the other and it must be proved that one is not the metastasis of the other [1,2]. When assessed according to these criteria, our case was categorized as a urinary 


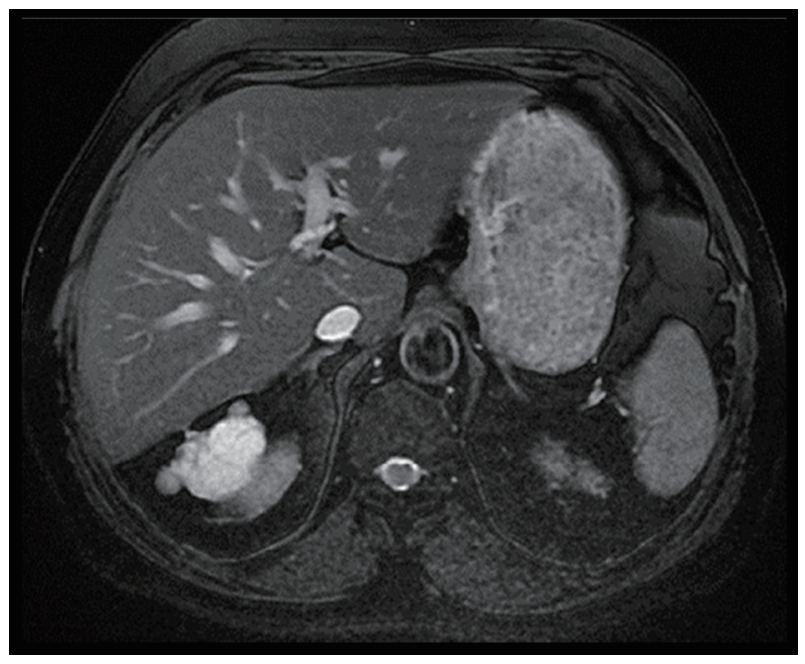

Figure 1. On axial fatty print T2A images, an exophytic placed cystic mass with a lobulated contour, and containing thick irregular septations and is $53 \times 22 \mathrm{~mm}$ on its widest part.

system cancer with synchrone. Our patient had MCRCC in his kidneys and high-grade urothelial carcinoma in his bladder. For every malignancy, there were several known predisposing factors. A cancer patient, depending on carcinogenic exposure such as tobacco and alcohol usage, genetic factors, side effects due to chemo-radiotherapy, carries a higher risk for other malignancies [3]. For our patient, the prominent one was tobacco usage. In addition, developments in diagnostic tools and increase in their use, and scanning other parts when one lesion was spotted had increased the percentage of identified cases with multiple tumors [2]. Two different tumors occurring on a patient simultaneously worsen the prognosis. The prognosis of a patient with dual malignancy depends on the more aggressive one of these tumors $[4,5]$. In such a case, the tumors' biological patterns, stages and the patient's concomitant diseases are all important factors affecting the treatment strategy [3]. Amongst urinary system tumors, the one with the highest rate of cooccurrence with a second malignancy is primary renal

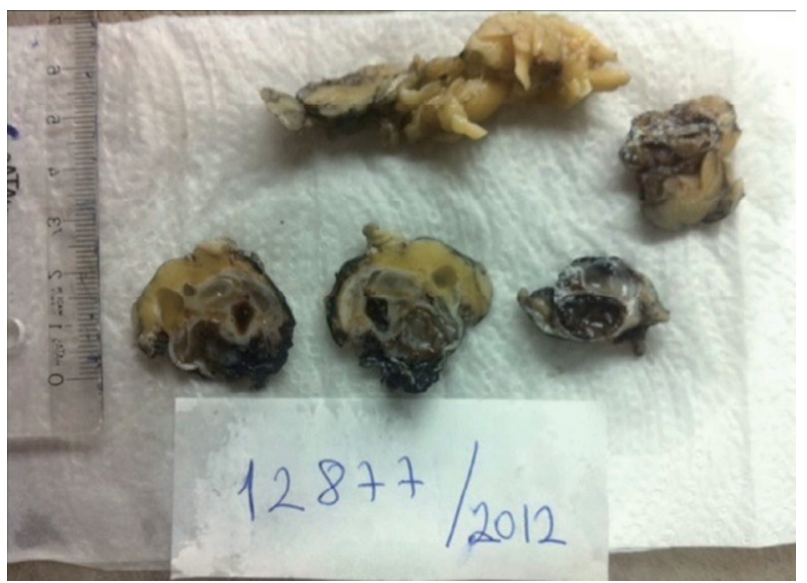

Figure 3. Many cystic formations that are $0.4-1 \mathrm{~cm}$ in size in the partial nephrectomy material.

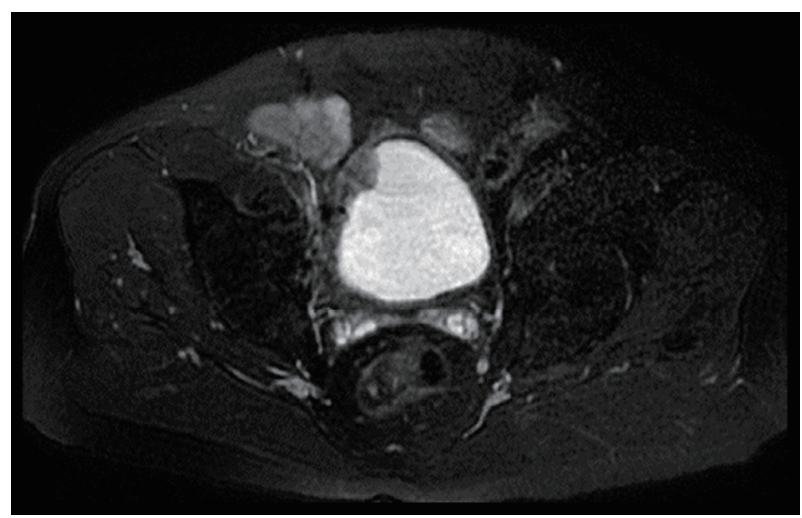

Figure 2. On axial fatty print T2A images, on the $2 \mathrm{~cm}$ thick bladder wall, a tumoral increase in wall thickness that is $1.5 \mathrm{~cm}$ on its thickest part.

cell carcinoma (17\%) [2]. A study shows that the second malignancies occurring in patients with primary RCC are for men prostate, bladder, colon, and rectum $\mathrm{Ca}$, and for women breast, bladder and gynecological tumors [2]. Our case was diagnosed with MCRCC and high-grade urothelial carcinoma of the bladder. RCC constitutes $3 \%$ of all adult tumors. MCRCC is a rare subtype of RCC and its prognosis is good. In the literature, its incidence is quiet low amongst other renal tumors. Robinson first diagnosed it in 1957. The diagnostic criteria for MCRCC first defined by Eble and Bonsib in 1998 were as such: 1) There must be an expansile mass surrounded by a fibrous capsule. 2) The interior of the tumor should completely consist of cysts and septas. Expansile nodule or solid component should be smaller than $10 \%$ of the tumor. 3) Septas should contain clear cell groups $[6,7]$. Although most RCCs originate from proximal tubules, a detailed immunohistochemical study performed by Imura et al has shown that MCRCC originates from distal tubules [8]. However, MCRCC's pathology still has not been completely illuminated [9]. In our case, as well, we observed that the tumor was surrounded by a fibrous capsule, and that it consisted of cysts that were divided by septas containing clear cell groups, and that the solid component was $2 \%$. According

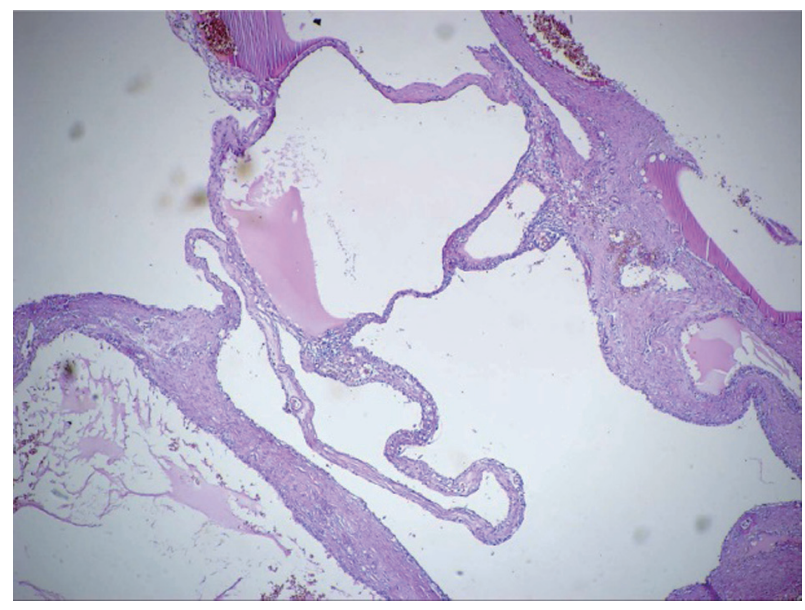

Figure 4. Multicystic appearance (H\&E, × 4). 


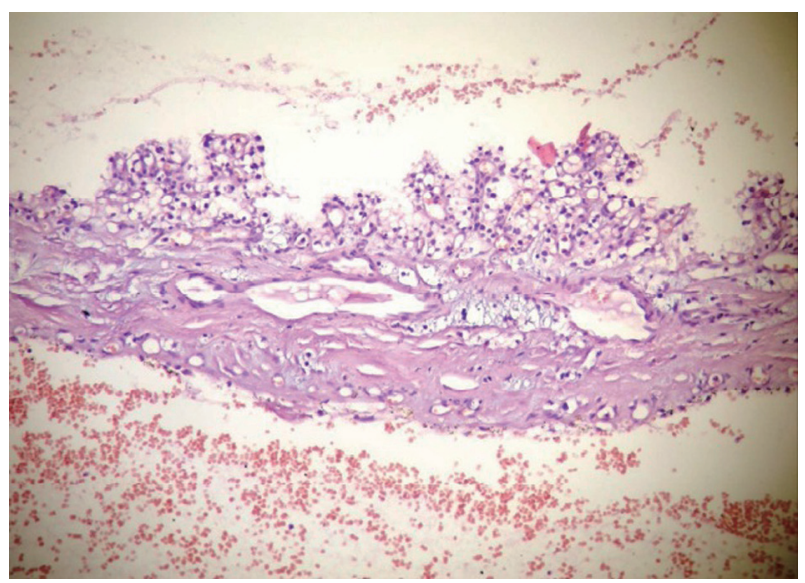

Figure 5. Small papillary formations formed by tumor cells with clear cytoplasm (H\&E, × 20).

to these findings, the diagnosis was MCRCC, and the stage was assessed as pT1. Urothelial carcinomas constitute $3.2 \%$ of all adult tumors. The stage and grade of the tumor are factors that affect prognosis. Depth of invasion is the most important factor that affects prognosis. For high graded tumors with muscle invasion, reoccurrence rate is $80 \%$, progression rate is $60 \%$, and 10 -year survival rate is $35 \%$ [10]. TUR BT material taken from our patient had a high-grade urothelial carcinoma that infiltrated muscle tissue. Although there are reports of synchronized kidney and bladder tumor cooccurrence in the literature, high-grade tumor of the bladder co-occurring with MCRCC, which is quiet rare as it is, has never been reported. Our case is the first one reporting these two tumors' cooccurrence.

\section{Conflict of Interest}

We declare that there is no conflict of interests regarding the publication of this article and that we do not have a direct financial or personal relation with any people or organizations.

\section{References}

1. Warren S, Gates O. Multiple primary malignant tumours: A survey of the literature and a statistical study. Am J Cancer. 1932;16:1358-1414.

2. Antonelli A, Calza S, Arrighi N, Zani D, Corti S, Cozzoli A, Zanotelli $\mathrm{T}$, et al. Clinical features and prognosis of patients with renal cancer and a second malignancy. Urol Oncol. 2012;30(3):294-300.

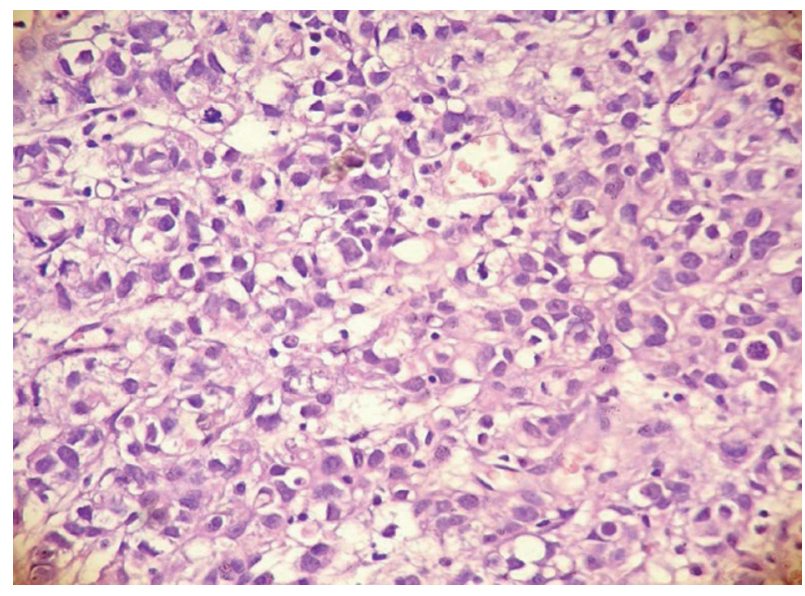

Figure 6. High-grade urothelial carcinoma consisting of cells with pleomorphic, large, atypical nucleus and eosinophilic cytoplasm and that has a high number of mitosis (H\&E, $\times 40)$.

3. Tiwari P, Tripathi A, Bansal P, Vijay M, Gupta A, Kundu AK. Synchronous primary cancers of urinary bladder and kidney and prostate. Saudi J Kidney Dis Transpl. 2012;23(4):786-789.

4. Dutta G, Silver D, Oliff A, Harrison A. Synchronous renal malignancy presenting as recurrent urinary tract infections. Case Rep Urol. 2011;2011:832673.

5. Leveridge M, Isotalo PA, Boag AH, Kawakami J. Synchronous ipsilateral renal cell carcinoma and urothelial carcinoma of the renal pelvis. Can Urol Assoc J. 2009;3(1):64-66.

6. Singhai A, Babu S, Verma N, Singh V. Multilocular cystic renal cell carcinoma: a rare entity. BMJ Case Rep. 2013;2013.

7. Eble JN, Bonsib SM. Extensively cystic renal neoplasms: cystic nephroma, cystic partially differentiated nephroblastoma, multilocular cystic renal cell carcinoma, and cystic hamartoma of renal pelvis. Semin Diagn Pathol. 1998;15(1):2-20.

8. Imura J, Ichikawa $\mathrm{K}$, Takeda J, Tomita S, Yamamoto H, Nakazono M, Takimoto T, et al. Multilocular cystic renal cell carcinoma: a clinicopathological, immunoand lectin histochemical study of nine cases. APMIS. 2004;112(3):183-191.

9. Cheong MS, Koo DH, Kim IS, Moon KC, Ku JH. Concurrent Multilocular Cystic Renal Cell Carcinoma and Leiomyoma in the Same Kidney: Previously Unreported Association. Case Rep Oncol. 2010;3(2):218-222.

10. WHO pathology and genetics tumors of the urinary system and male genital organs 2004. 